PROCEEDINGS OF THE

AMERICAN MATHEMATICAL SOCIETY

Volume 125, Number 8, August 1997, Pages 2217-2219

S $0002-9939(97) 03917-8$

\title{
LIFTING OF GENERATING SUBGROUPS
}

\author{
IDO EFRAT
}

(Communicated by Ronald M. Solomon)

\begin{abstract}
Let $\varphi: G \rightarrow H$ be an epimorphism of finite groups. Suppose that $G$ is generated by its subgroups $G_{1}, \ldots, G_{n}$ and that $H$ is generated by its subgroups $H_{1}, \ldots, H_{n}$. Furthermore, suppose that $\varphi\left(G_{i}\right)$ and $H_{i}$ are conjugate, $i=1, \ldots, n$. We prove that there exist $g_{1}, \ldots, g_{n} \in G$ such that $G_{1}^{g_{1}}, \ldots, G_{n}^{g_{n}}$ generate $G$ and $\varphi\left(G_{i}^{g_{i}}\right)=H_{i}, i=1, \ldots, n$.
\end{abstract}

In this note we prove the following lifting principle for generating subgroups:

Main Theorem. Let $\varphi: G \rightarrow H$ be an epimorphism of finite groups and let $G_{1}, \ldots, G_{n}$ and $H_{1}, \ldots, H_{n}$ be subgroups of $G$ and $H$, respectively. Suppose that $G=\left\langle G_{1}, \ldots, G_{n}\right\rangle$ and $H=\left\langle H_{1}, \ldots, H_{n}\right\rangle$. Moreover, suppose that $\varphi$ maps some conjugate of $G_{i}$ onto $H_{i}, i=1, \ldots, n$. Then there exist conjugates $G_{1}^{g_{1}}, \ldots, G_{n}^{g_{n}}$ of $G_{1}, \ldots, G_{n}$ which are mapped by $\varphi$ onto $H_{1}, \ldots, H_{n}$, respectively, and which generate $G$.

This theorem and its proof are motivated by the following result:

Theorem (Gaschütz [4]). Let $\varphi: G \rightarrow H$ be an epimorphism of finite groups and let $\left\{h_{1}, \ldots, h_{n}\right\}$ be a set of generators for $H$. Suppose that $G$ can be generated by $n$ elements. Then there exists a set $\left\{g_{1}, \ldots, g_{n}\right\}$ of generators for $G$ such that $\varphi\left(g_{i}\right)=h_{i}, i=1, \ldots, n$.

Gaschütz proved this fact by means of a sophisticated counting argument. His proof has later been simplified by Roquette [2, Lemma 15.30]. This theorem had an important and quite surprising role in the model theory of fields [3]. See also [1] for a generalization of Gaschütz' theorem, which however does not cover our main theorem.

Proof of the Main Theorem. Let $J$ be the collection of all subgroups $F$ of $G$ such that $\varphi(F)=H$. For $F \in J, h \in H$ and a left coset $g F \in G / F$ (where $g \in G$ ) let

$$
A_{i}(F, h, g F)=g F \cap \varphi^{-1}\left(N_{H}\left(\varphi\left(G_{i}\right)\right) h\right), \quad i=1, \ldots, n,
$$

where $N_{H}$ denotes the normalizer of a subgroup of $H$. Thus $g^{\prime} \in A_{i}(F, h, g F)$ if and only if $g$ and $g^{\prime}$ belong to the same left coset in $G / F$ and $\varphi\left(G_{i}^{g^{\prime}}\right)=\varphi\left(G_{i}\right)^{h}$. We show that the size of the finite set $A_{i}(F, h, g F)$ does not depend on the choice of $h$. Indeed, for any other $h^{\prime} \in H$ we can take $f, f^{\prime} \in F$ with $\varphi(f)=h$ and $\varphi\left(f^{\prime}\right)=h^{\prime}$. Then $A_{i}\left(F, h^{\prime}, g F\right)=A_{i}(F, h, g F) f^{-1} f^{\prime}$.

Received by the editors February 13, 1996.

1991 Mathematics Subject Classification. Primary 20D99.

(C)1997 American Mathematical Society 
For $F \in J$, for $h_{1}, \ldots, h_{n} \in H$, and for left cosets $g_{1} F, \ldots, g_{n} F \in G / F$ (where $\left.g_{1}, \ldots, g_{n} \in G\right)$ set:

$$
\begin{aligned}
B\left(F, h_{1}, \ldots, h_{n}, g_{1} F, \ldots, g_{n} F\right) & \\
= & \left\{\left(g_{1}^{\prime}, \ldots, g_{n}^{\prime}\right) \in \prod_{i=1}^{n} A_{i}\left(F, h_{i}, g_{i} F\right) \mid F=\left\langle G_{1}^{g_{1}^{\prime}}, \ldots, G_{n}^{g_{n}^{\prime}}\right\rangle\right\} .
\end{aligned}
$$

Claim. Let $F \in J$, let $h_{1}, \ldots, h_{n} \in H$ satisfy $H=\left\langle\varphi\left(G_{1}\right)^{h_{1}}, \ldots, \varphi\left(G_{n}\right)^{h_{n}}\right\rangle$, and let $g_{i} \in G$ satisfy $G_{i}^{g_{i}} \leq F, i=1, \ldots, n$. Then:

$$
\begin{aligned}
& \prod_{i=1}^{n} A_{i}\left(F, h_{i}, g_{i} F\right)=\bigcup \mathcal{S}\left\{B\left(F^{*}, h_{1}, \ldots, h_{n}, g_{1}^{\prime} F^{*}, \ldots, g_{n}^{\prime} F^{*}\right)\right. \mid F^{*} \in J, \\
&\left.F^{*} \leq F, g_{i}^{\prime} F=g_{i} F, i=1, \ldots, n\right\} .
\end{aligned}
$$

Indeed, it is straightforward to check that the right-hand side is contained in the left-hand side. Conversely, suppose that $\left(g_{1}^{\prime}, \ldots, g_{n}^{\prime}\right) \in \prod_{i=1}^{n} A\left(F, h_{i}, g_{i} F\right)$ and set $F^{*}=\left\langle G_{1}^{g_{1}^{\prime}}, \ldots, G_{n}^{g_{n}^{\prime}}\right\rangle$. Clearly, $\left(g_{1}^{\prime}, \ldots, g_{n}^{\prime}\right) \in B\left(F^{*}, h_{1}, \ldots, h_{n}, g_{1}^{\prime} F^{*}, \ldots, g_{n}^{\prime} F^{*}\right)$. In addition, $g_{i}^{\prime} F=g_{i} F$ and $G_{i}^{g_{i}} \leq F$, so one has $G_{i}^{g_{i}^{\prime}} \leq F$ for each $1 \leq i \leq n$, whence $F^{*} \leq F$. Also $g_{i}^{\prime} \in A_{i}\left(F, h_{i}, g_{i} F\right)$ implies that $\varphi\left(G_{i}^{g_{i}^{\prime}}\right)=\varphi\left(G_{i}\right)^{h_{i}}$, and we obtain:

$$
\varphi\left(F^{*}\right)=\left\langle\varphi\left(G_{1}^{g_{1}^{\prime}}\right), \ldots, \varphi\left(G_{n}^{g_{n}^{\prime}}\right)\right\rangle=\left\langle\varphi\left(G_{1}\right)^{h_{1}}, \ldots, \varphi\left(G_{n}\right)^{h_{n}}\right\rangle=H .
$$

Therefore $F^{*} \in J$. Conclude that $\left(g_{1}^{\prime}, \ldots, g_{n}^{\prime}\right)$ belongs to the right-hand side.

To see that this union is disjoint we need to prove that an $n$-tuple

$$
\left(g_{1}^{\prime \prime}, \ldots, g_{n}^{\prime \prime}\right) \in B\left(F^{*}, h_{1}, \ldots, h_{n}, g_{1}^{\prime} F^{*}, \ldots, g_{n}^{\prime} F^{*}\right)
$$

already determines $F^{*}$ and $g_{1}^{\prime} F^{*}, \ldots, g_{n}^{\prime} F^{*}$. Now $F^{*}=\left\langle G_{1}^{g_{1}^{\prime \prime}}, \ldots, G_{n}^{g_{n}^{\prime \prime}}\right\rangle$, and as $g_{i}^{\prime \prime} \in A_{i}\left(F^{*}, h_{i}, g_{i}^{\prime} F^{*}\right)$ we have $g_{i}^{\prime} F^{*}=g_{i}^{\prime \prime} F^{*}, i=1, \ldots, n$. This proves the claim.

By the assumption of the theorem there exist $h_{1}, \ldots, h_{n} \in H$ with $\varphi\left(G_{i}\right)^{h_{i}}=H_{i}$, $i=1, \ldots, n$. Therefore

$$
H=\left\langle\varphi\left(G_{1}\right)^{h_{1}}, \ldots, \varphi\left(G_{n}\right)^{h_{n}}\right\rangle
$$

as well as

$$
H=\varphi(G)=\left\langle\varphi\left(G_{1}\right), \ldots, \varphi\left(G_{n}\right)\right\rangle .
$$

By the first part of the proof, $\left|A_{i}\left(F, h_{i}, g_{i} F\right)\right|=\left|A_{i}\left(F, 1, g_{i} F\right)\right|$ for every $F \in J$ and cosets $g_{i} F \in G / F, i=1, \ldots, n$. Assuming in addition that $G_{i}^{g_{i}} \leq F$ for each $i$, the claim implies that

$$
\begin{aligned}
& \left|B\left(F, h_{1}, \ldots, h_{n}, g_{1} F, \ldots, g_{n} F\right)\right|+\sum\left|B\left(F^{*}, h_{1}, \ldots, h_{n}, g_{1}^{\prime} F^{*}, \ldots, g_{n}^{\prime} F^{*}\right)\right| \\
= & \prod_{i=1}^{n}\left|A_{i}\left(F, h_{i}, g_{i} F\right)\right|=\prod_{i=1}^{n}\left|A_{i}\left(F, 1, g_{i} F\right)\right| \\
= & \left|B\left(F, 1, \ldots, 1, g_{1} F, \ldots, g_{n} F\right)\right|+\sum\left|B\left(F^{*}, 1, \ldots, 1, g_{1}^{\prime} F^{*}, \ldots, g_{n}^{\prime} F^{*}\right)\right|
\end{aligned}
$$

where the sums are taken over all proper subgroups $F^{*}$ of $F$ which belong to $J$ and over all left cosets $g_{1}^{\prime} F^{*}, \ldots, g_{n}^{\prime} F^{*}$ in $G / F^{*}$ satisfying $g_{i}^{\prime} F=g_{i} F, i=1, \ldots, n$. 
Note that when $g_{i}^{\prime} F=g_{i} F$ one has $G_{i}^{g_{i}^{\prime}} \leq F$ as well, $i=1, \ldots, n$. By induction on the size of $F \in J$ we now obtain that

$$
\left|B\left(F, h_{1}, \ldots, h_{n}, g_{1} F, \ldots, g_{n} F\right)\right|=\left|B\left(F, 1, \ldots, 1, g_{1} F, \ldots, g_{n} F\right)\right|
$$

for every $F \in J$ and $g_{i} F \in G / F$ which satisfy $G_{i}^{g_{i}} \leq F, i=1, \ldots, n$. Since $(1, \ldots, 1) \in B(G, 1, \ldots, 1, G, \ldots, G)$ also $B\left(G, h_{1}, \ldots, h_{n}, G, \ldots, G\right) \neq \emptyset$. But for every $n$-tuple $\left(g_{1}^{\prime}, \ldots, g_{n}^{\prime}\right)$ in the latter set we have $G=\left\langle G_{1}^{g_{1}^{\prime}}, \ldots, G_{n}^{g_{n}^{\prime}}\right\rangle$ as well as

$$
\varphi\left(G_{i}^{g_{i}^{\prime}}\right)=\varphi\left(G_{i}\right)^{h_{i}}=H_{i},
$$

$i=1, \ldots, n$. This gives conjugates of $G_{1}, \ldots, G_{n}$ as required.

\section{REFERENCES}

1. I. Efrat, The elementary theory of free pseudo p-adically closed fields of finite corank, J. Sym. Logic 56 (1991), 484-496. MR 93a:03036

2. M. Fried and M. Jarden, Field Arithmetic, Springer, Heidelberg, 1986. MR 89b:12010

3. M. Jarden and U. Kiehne, The elementary theory of algebraic fields of finite corank, Inventiones Math. 30 (1975), 275-294. MR 55:8012

4. W. Gaschütz, Zu einem von B.H. und H. Neumann gestellten Problem, Math. Nachr. 14 (1956), 249-252. MR 18:790b

Department of Mathematics and Computer Science, Ben-Gurion University of the Negev, P.O. Box 653, Be'er-Sheva 84105, Israel

E-mail address: efrat@math.bgu.ac.il 